\title{
RESEARCH
}

Open Access

\section{The angiogenic potential of CD271+ human adipose tissue-derived mesenchymal stem cells}

\author{
Richard J. P. Smith", Alessandro Faroni', James R. Barrow ${ }^{1}$, Jamie Soul ${ }^{2}$ and Adam J. Reid ${ }^{1,3^{*}}$ (D)
}

\begin{abstract}
Background: Autologous fat grafting is often a crucial aspect of reconstructive and aesthetic surgeries, yet poor graft retention is a major issue with this technique. Enriching fat grafts with adipose tissue-derived mesenchymal stem cells (AD-MSCs) improves graft survival-however, AD-MSCs represent a heterogeneous population. Selection of subpopulations of AD-MSCs would allow the targeting of specific AD-MSCs that may benefit fat graft survival more than the general AD-MSC population.

Methods: Human AD-MSCs were selected for the surface marker CD271 using magnetic-activated cell sorting and compared to the CD271 negative phenotype. These subpopulations were analysed for gene expression using RealTime QPCR and RNA sequencing; surface marker characteristics using immunostaining; ability to form tubules when cultured with endothelial cells; and gene and protein expression of key angiogenic mediators when cultured with ex-vivo adipose tissue.
\end{abstract}

Results: Human AD-MSCs with the surface marker CD271 express angiogenic genes at higher levels, and inflammatory genes at lower levels, than the CD271- AD-MSC population. A greater proportion of CD271+ ADMSCs also possess the typical complement of stem cell surface markers and are more likely to promote effective neoangiogenesis, compared to CD271- AD-MSCS.

Conclusion: Enriching grafts with the CD271+ AD-MSC subpopulation holds potential for the improvement of reconstructive and aesthetic surgeries involving adipose tissue.

Keywords: Adipose tissue-derived mesenchymal stem cells (AD-MSCS), Stromal vascular fraction (SVF), CD271, Angiopoietin, Fat grafting, Magnetic-activated cell sorting (MACS), Adipose tissue engineering

\footnotetext{
* Correspondence: adam.reid@manchester.ac.uk

'Blond McIndoe Laboratories, Division of Cell Matrix Biology and

Regenerative Medicine, School of Biological Sciences, Faculty of Biology,

Medicine and Health, The University of Manchester, Manchester Academic

Health Science Centre, Manchester M13 9PT, UK

${ }^{3}$ Department of Plastic Surgery \& Burns, Wythenshawe Hospital, Manchester

University NHS Foundation Trust, Manchester Academic Health Science

Centre, Manchester, UK

Full list of author information is available at the end of the article
}

(c) The Author(s). 2021 Open Access This article is licensed under a Creative Commons Attribution 4.0 International License, which permits use, sharing, adaptation, distribution and reproduction in any medium or format, as long as you give appropriate credit to the original author(s) and the source, provide a link to the Creative Commons licence, and indicate if changes were made. The images or other third party material in this article are included in the article's Creative Commons licence, unless indicated otherwise in a credit line to the material. If material is not included in the article's Creative Commons licence and your intended use is not permitted by statutory regulation or exceeds the permitted use, you will need to obtain permission directly from the copyright holder. To view a copy of this licence, visit http://creativecommons.org/licenses/by/4.0/ The Creative Commons Public Domain Dedication waiver (http://creativecommons.org/publicdomain/zero/1.0/) applies to the data made available in this article, unless otherwise stated in a credit line to the data. 


\section{Background}

Human adipose tissue-derived mesenchymal stem cells (AD-MSCs) have been investigated as a therapeutic cell population for a number of potential clinical applications including autologous fat transplantation or fat grafting [1]. The addition of autologous cell therapy has been investigated in an effort to improve tissue survival during fat grafting, with mixed success [2]. The stromal vascular fraction (SVF), easily derived from human adipose tissue (AT), contains AD-MSCs, among many other cell types, and exhibits regenerative potential for various indications including burns, nerve injury, and fractures [3]. Underpinning this potential, AD-MSCs have been shown to release several growth factors in vitro, including the angiogenic markers hepatocyte growth factor (HGF), transforming growth factor beta (TGF- $\beta$ ), and vascular endothelial growth factor A (VEGFA), at levels that assist recovery from ischemia in mice and could potentially improve tissue survival [4]. Accordingly, cultured AD-MSCs have been shown to positively influence in vitro endothelial tubule formation via microvesicle signalling [5].

The highly heterogeneous nature of the SVF means this cell population could be refined for particular clinical applications. Endothelial, immune, and blood cells are unlikely to provide support to a de-vascularised adipose tissue graft, and death of these cells in the graft may counter the trophic support of AD-MSCs [6, 7]. As such, we propose that a more targeted therapeutic approach is required, whereby subpopulations of cells from the SVF are selected for their angiogenic characteristics.

Previous studies have highlighted CD271+ AD-MSCs as a subpopulation of interest for therapeutic application. Also known as low-affinity nerve growth factor receptor (LNGFR), or p75, CD271 is a receptor found highly expressed in the glial cells of the central and peripheral nervous systems [8, 9]. CD271 has for many years been a popular target for the isolation of bone marrowderived mesenchymal stem cells (BM-MSCs), as selection for CD271 effectively eliminates endothelial and haematopoietic cells from the BM-MSC population [10]. AD-MSCs expressing CD271 have been found to also express Notch1, Rex1, and Nestin, markers of self-renewal, pluripotency, and cytoskeletal competency respectively [11-13]. In human AT, the expression of CD271 is variable between patients, but typically between 4 and $20 \%$ of the extracted AD-MSC population expresses CD271, as measured by flow cytometry [14-16]. Although CD271 expression in AD-MSCs in vivo has been shown to decrease with age, the expression is still higher than that found in BM-MSCs [17].

Here, we show that CD271+ AD-MSCs hold great potential in tissue engineering applications. CD271+ ADMSCs exhibit increased expression of angiogenic markers, are more likely to belong to the typical MSC phenotype CD45-/CD90+, produce more complex vascular networks, and contribute to a more angiogenic environment when grown with $\mathrm{AT}$, compared to CD271AD-MSCs.

\section{Materials and methods}

\section{Adipose tissue sourcing and harvest}

Abdominal adipose tissue was harvested from donor patients undergoing DIEP flap breast reconstruction at Wythenshawe Hospital, Manchester University NHS Foundation Trust, UK, after informed consent was granted. Ethics approval was given from the NHS Health Research Authority, National Research Ethics Service Committee South Central - Hampshire B: reference 13/ $\mathrm{SC} / 0499$. Fresh adipose tissue was minced into a homogenous pulp using surgical scissors and razor blades. The pulp was divided into 50-ml falcon tubes with an equal volume of $0.2 \%(\mathrm{w} / \mathrm{v})$ collagenase I in HBSS and agitated in a $37^{\circ} \mathrm{C}$ water bath for $1 \mathrm{~h}$. The resulting digested tissue was filtered through a $100-\mu \mathrm{m}$ nylon mesh (Merck Millipore UK Ltd., Watford, UK), and to this was added an equal volume of $\alpha$-Minimum Essential Medium Eagle ( $\alpha$ MEM, Sigma Aldrich, Poole, UK, always containing unless otherwise stated: 10\% Foetal Bovine Serum (FBS, LabTech, Uckfield, UK), 1\% L-Glutamine (GE Healthcare UK Ltd., Little Chalfont, UK), $1 \%$ PenStrep). The tubes were centrifuged at $160 g$ for $10 \mathrm{~min}$. The pellet (the stromal vascular fraction, SVF) was resuspended in $1 \mathrm{ml}$ Red Blood Cell Lysis Buffer (Sigma Aldrich, Poole, UK) for $1 \mathrm{~min}$; then, $20 \mathrm{ml}$ aMEM was added to arrest lysis. The mixture was centrifuged at $160 \mathrm{~g}$ for $10 \mathrm{~min}$, and the resulting pellet was resuspended in freezing mix (FBS $+10 \%$ DMSO (dimethyl sulfoxide, Sigma Aldrich, Poole, UK)) and slowfrozen to $-80^{\circ} \mathrm{C}$ until further use.

\section{Magnetic-activated cell sorting (MACS)}

Frozen SVF was thawed in a $37^{\circ} \mathrm{C}$ water bath and resuspended in $10 \mathrm{ml}$ aMEM. The suspension was passed through a 40- $\mu \mathrm{m}$ cell strainer which was washed with an additional $10 \mathrm{ml}$ aMEM. The cells were counted (Scepter 2.0 automated cell counter, Merck Millipore UK Ltd., Watford, UK) and around 1\% of total cells were set aside as the unsorted population. The remaining suspension was centrifuged at $300 \mathrm{~g}$ for $10 \mathrm{~min}$, and the cell pellet resuspended in $60 \mu \mathrm{l}$ MACS buffer $(0.5 \%$ Bovine Serum Albumin (BSA, Sigma Aldrich, Poole, UK) and $2 \mathrm{mM}$ Ethylenediaminetetraacetic Acid (EDTA, Sigma Aldrich, Poole, UK) in Phosphate Buffer Saline solution (PBS), $20 \mu \mathrm{l}$ CD271 microbead solution (Miltenyi Biotec, Surrey, UK, 130-099-023), and $20 \mu \mathrm{l} \mathrm{FcR} \mathrm{blocking} \mathrm{reagent}$ (Miltenyi Biotec, Surrey, UK, 130-099-023) per $10^{7}$ cells and incubated for $15 \mathrm{~min}$ at $4{ }^{\circ} \mathrm{C}$. The cells were then washed with $1 \mathrm{ml}$ MACS buffer per $10^{7}$ cells and 
centrifuged at $300 \mathrm{~g}$ for $10 \mathrm{~min}$ and resuspended in MACS buffer. The magnetic column (Miltenyi Biotec, Surrey, UK, 130-042-401) was attached to a MACS magnet (Miltenyi Biotec, Surrey, UK, 130-090-976), and the cell suspension was passed through the column, with flow-through collected as the negative population (CD271- AD-MSCs). Subsequently, the column was removed from the magnet, and the remaining cells flushed out of the column using the plunger: this was the positive population (CD271+ AD-MSCs). At least 200,000 cells for each group were used for flow cytometry analysis.

\section{Flow cytometry}

Cells from the sorting procedure were centrifuged at $300 \mathrm{~g}$ for $10 \mathrm{~min}$ and pellets resuspended in the appropriate fluorescent antibodies or isotype control antibodies at a concentration of 1:11 in MACS buffer. Cells were incubated in up to three antibodies for $10 \mathrm{~min}$ at $4{ }^{\circ} \mathrm{C}$. After a final wash step, cells were resuspended in MACS buffer and transported directly for flow cytometry and analysed for surface marker expression using a Cyan ADP flow cytometer (Beckman Coulter, High Wycombe, UK) at the Faculty of Biology, Medicine and Health Core Facility, University of Manchester. Compensation was carried out using a bead kit (Miltenyi Biotec, Surrey, UK, 130-097-900). All antibodies and isotype controls used were obtained from Miltenyi Biotech (Surrey, UK) and are as follows with product codes: CD29-PE (130-101275), CD34-PE-Vio770 (130-100-844), CD45-PerCP (130-098-145), CD90-FITC (130-097-930), CD146VioBlue (130-099-678), CD271-APC (130-091-884), Mouse IgG1-PE (130-098-845), Mouse IgG2a-PE-Vio770 (130-098-564), Mouse IgG2a-PerCP (130-099-190), Mouse IgG1-FITC (130-098-847), Mouse IgG1-VioBlue (130-099-756), and Mouse IgG1-APC (130-098-846). Data were analysed using FlowJo v10 (FlowJo LLC, Ashland, OR, USA).

\section{Human AD-MSC culture}

Both sorted and unsorted AD-MSCs were cultured in the same manner, in $\mathrm{T} 75$ flasks at $37^{\circ} \mathrm{C}, 5 \% \mathrm{CO}_{2}$, with three media changes of $10-\mathrm{ml}$ fresh $\alpha M E M$ every week. The protocol for passaging cells was as follows: HBSS wash, $5 \mathrm{~min}$ in $3 \mathrm{ml} 0.25 \%$ Trypsin (Life Technologies Ltd., Paisley, UK), addition of $5 \mathrm{ml} \alpha \mathrm{MEM}$, centrifugation at $900 \mathrm{rpm}$ for $5 \mathrm{~min}$, resuspension of pellet in QMEM, and replating. Observation of cultured ADMSCs was achieved using a light microscope (Olympus IX51).

\section{Immunocytochemistry}

Staining for CD271 and CD31 was carried out on tissue fixed with paraformaldehyde (PFA). Immediately upon receipt of human tissue, small $0.5-\mathrm{cm}^{3}$ chunks of tissue were incubated in $4 \%$ PFA for $24 \mathrm{~h}$, before being washed three times with PBS-Sucrose for $24 \mathrm{~h} /$ wash. Tissue was then embedded in OCT matrix (Fischer Scientific, Loughborough, UK) and flash-frozen with liquid nitrogen. Tissue was cryosectioned onto glass slides in 30- $\mu \mathrm{m}$ slices and dried at $37{ }^{\circ} \mathrm{C}$ overnight. Slides were rehydrated in PBS for 10 min before being washed in $0.2 \%$ Triton X-100 in PBS for $1 \mathrm{~h}$. Slides were blocked with the appropriate serum (Normal Goat Serum 1:100 (Sigma Aldrich, Poole, UK) in antibody diluent (1\% BSA, 1\% Sodium Azide, $0.3 \%$ Triton X-100 in PBS)) for $1 \mathrm{~h}$ at room temperature, washed with $\mathrm{PBS}$, then incubated in primary antibodies overnight: rabbit monoclonal antiCD271 antibody (Abcam, UK, ab52987) and mouse monoclonal anti-CD31 antibody (Abcam, UK, ab24590) diluted 1:100 in antibody diluent. Next, slides were washed in PBS and secondary antibody was added (Goat anti-rabbit 488, Thermo Fisher Scientific, UK, A27034, and Donkey anti-mouse 568, Thermo Fisher Scientific, UK, A10037) at 1:500 in antibody diluent. After 2-h incubation at room temperature, and a PBS wash, coverslips were fixed with DAPI-containing Vectashield (Vector Labs, UK, H-1200) to stain for cell nuclei. Slides were viewed under a fluorescent microscope (Olympus BX60) and images recorded in Image Pro Plus (v9.1, Media Cybernetics).

\section{Real-time qPCR and RNA sequencing}

RNA was extracted from sorted and differentiated ADMSCs using a Qiagen RNeasy plus mini kit (Qiagen, Manchester, UK), and RNA was extracted from frozen adipocytes using a Qiagen RNeasy lipid tissue mini kit (Qiagen, Manchester, UK). cDNA was transcribed from RNA using Qiagen RT2 reverse transcription kit (Qiagen, Manchester, UK). Between 1 and 3 ng cDNA was used in each reaction, kept consistent within each experimental run. The PCR mix within each individual reaction consisted of $1 \mu \mathrm{l}$ of sample, along with $12.5 \mu \mathrm{l} \mathrm{RT}{ }^{2}$ SYBR green mastermix (Qiagen, Manchester, UK), $1 \mu \mathrm{l}$ primer mix (primers shown in Supplemental Table 1) and made up to $25 \mu \mathrm{l}$ total with RNase-free water. Samples were run in a Corbett Rotor-Gene 6000 with the following protocol: $10 \mathrm{~min}$ at $95^{\circ} \mathrm{C}, 45$ cycles of $15 \mathrm{~s}$ at $95^{\circ} \mathrm{C}, 30 \mathrm{~s}$ at $60^{\circ} \mathrm{C}$ and $30 \mathrm{~s}$ at $72^{\circ} \mathrm{C}$, then hold at $95^{\circ} \mathrm{C}$ for $1 \mathrm{~min}, 65^{\circ} \mathrm{C}$ for $2 \mathrm{~min}$, then melting curve from 65 to $95^{\circ} \mathrm{C}$ at $2{ }^{\circ} \mathrm{C} / \mathrm{min}$. Analysis was performed in the RotorGene's analysis software using the $\Delta \Delta \mathrm{C}_{\mathrm{T}}$ method. Relative expression in individual samples was normalised to $18 \mathrm{~s}$, and changes between samples were normalised to either CD271- AD-MSCs (characteristics analysis, see the 'Results' section, 'Identification, extraction, and characterisation of CD271+ AD-MSCs') or CD271- AD- 
MSCs with AT (co-culture analysis, see the 'Results' section, 'Co-culture of CD271+ AD-MSCs with AT').

RNA sequencing was carried out with the assistance of the Bioinformatics core facility at the University of Manchester. Samples were sorted using MACS as above, then preserved in RNA Later (Qiagen, 76106), and immediately sent to the core facility for RNA extraction and analysis. Briefly, reads were trimmed with Trimmomatic v0.36, mapped to the reference genome using STAR v2.5.1a, ensEMBL v90, and Gencode v27 (freeze date 01/27, GRCh38). Gene count was achieved using HTSeq-count v0.6.1p1 and normalised using DESeq2 v1.10.0, allowing samples to be directly compared.

\section{Reactome pathway analysis}

Differentially expressed genes (1.5 absolute fold change and Benjamini-Hochberg-corrected $p$ values $\leq 0.05$ ) were used with the $\mathrm{R}$ package GOSeq to identify differentially regulated pathways. Pathways with BenjaminiHochberg-corrected $p$ values of $\leq 0.05$ were regarded as statistically significant $[18,19]$.

\section{HUVEC tubule formation assay}

Human umbilical vascular endothelial cells (HUVEC) were acquired from Merck Millipore (SCCE001) and plated according to instructions at 5000 cells per $\mathrm{cm}^{2}$ in T75 flasks. Cells were grown in standard culture conditions (see above). Media used was EndoGro-LS from Merck Millipore (SCME001). Upon reaching confluence, HUVEC cells were trypsinised and plated on 24-well plates coated with Matrigel, at a seeding density of 16 , 000 cells per well. Matrigel coating was achieved using a 1:1 dilution of Matrigel (Corning UK, 356234) in EndoGro-LS media. Once HUVEC cells were plated in the Matrigel-coated wells, AD-MSCs were sorted and seeded with the HUVEC cells at a density of 8000 cells per well. Cells were cultured in EndoGro-LS media for $16 \mathrm{~h}$, before images were recorded using light microscopy. The average tubule length was recorded using the Image J (v1.47f) 'measure' tool. Average tubule lengths were calculated, with at least ten images from each experimental group. Number of anastomoses was counted.

For co-staining of HUVECs and AD-MSCs, the above procedure for growing the cells together was followed, although with pre-labelled red fluorescent protein (RFP) expressing HUVEC obtained from Angio-Proteomie (cAP-0001RFP) at passage 3, and passage 3 CD271+ AD-MSCs stained with carboxyfluorescein succinimidyl ester (CFSE). Images were recorded using fluorescence microscopy.

\section{Ex vivo co-culture}

Following the protocol of Anayama et al., an ex vivo model of AD-MSC/adipose tissue co-culture was set up using special well inserts [20]. Immediately following rough tissue homogenisation with a razor blade, $0.1 \mathrm{ml}$ units of undigested adipose tissue were added to the centre of specialised well inserts (Merck Millipore, PICM03050) sitting in six-well plates. Two millilitres of media (1:1 F12:DMEM mix, 10\% FBS, 1\% Pen-strep) was added to each well, so that media reached the membrane of the inserts, but did not cover them. Remaining tissue was sorted using the standard MACS protocol. Sorted AD-MSCs were seeded in separate six-well plates at 150, 000 cells/well, and grown in the same F12:DMEM mix as the adipose tissue. After $48 \mathrm{~h}$, the inserts containing adipose tissue were transferred across to the wells containing AD-MSCs-this marked day 0 of the co-culture. Media was changed every 2 days, and cells cultured in normal conditions, with the L-glutamate-free F12: DMEM media mix, for 15 days. At the endpoint, ADMSCs were collected in RNA Later (Qiagen, 76106) for PCR analysis, and media was removed for ELISA.

\section{ELISA}

ELISA kits were purchased from RayBiotech for the analysis of levels of VEGF-A (product ELH-VEGF), HGF (product ELH-HGF), and angiopoietin-1 (product ELHANG1) in conditioned media. After 15 days of coculture, media was collected after $48 \mathrm{~h}$ of incubation. A standard curve of target protein was created for each kit, and media was added to the microplates in triplicate and incubated according to the kit's instructions. Absorbance was read at $450 \mathrm{~nm}$. To calculate protein concentrations, the standard curve was plotted in Graph Pad Prism (v6) and absorbances of unknown samples were interpolated against this curve to produce protein concentration values.

\section{Statistical analysis}

All statistical analysis was undertaken in Graph Pad Prism (v6). One-way or two-way ANOVAs with either Tukey's or Sidak's multiple comparisons tests were performed in all cases with more than two comparisons, and unpaired $t$ tests were carried out in other cases. All data are presented as means \pm SEM. In all cases, significance was assumed when $p<0.05$, and ${ }^{*} p<0.05,{ }^{* *} p<$ $0.01,{ }^{* * * *} p<0.001,{ }^{* * * * * *} p<0.0001$.

\section{Results}

Identification, extraction, and characterisation of CD271+ AD-MSCs

Staining of sections of human adipose tissue (AT) revealed that CD271 was found exclusively around vascular structures, confirmed by co-localisation with CD31 staining (Fig. 1), suggesting that CD271+ AD-MSCs are involved in vascular function in vivo. 

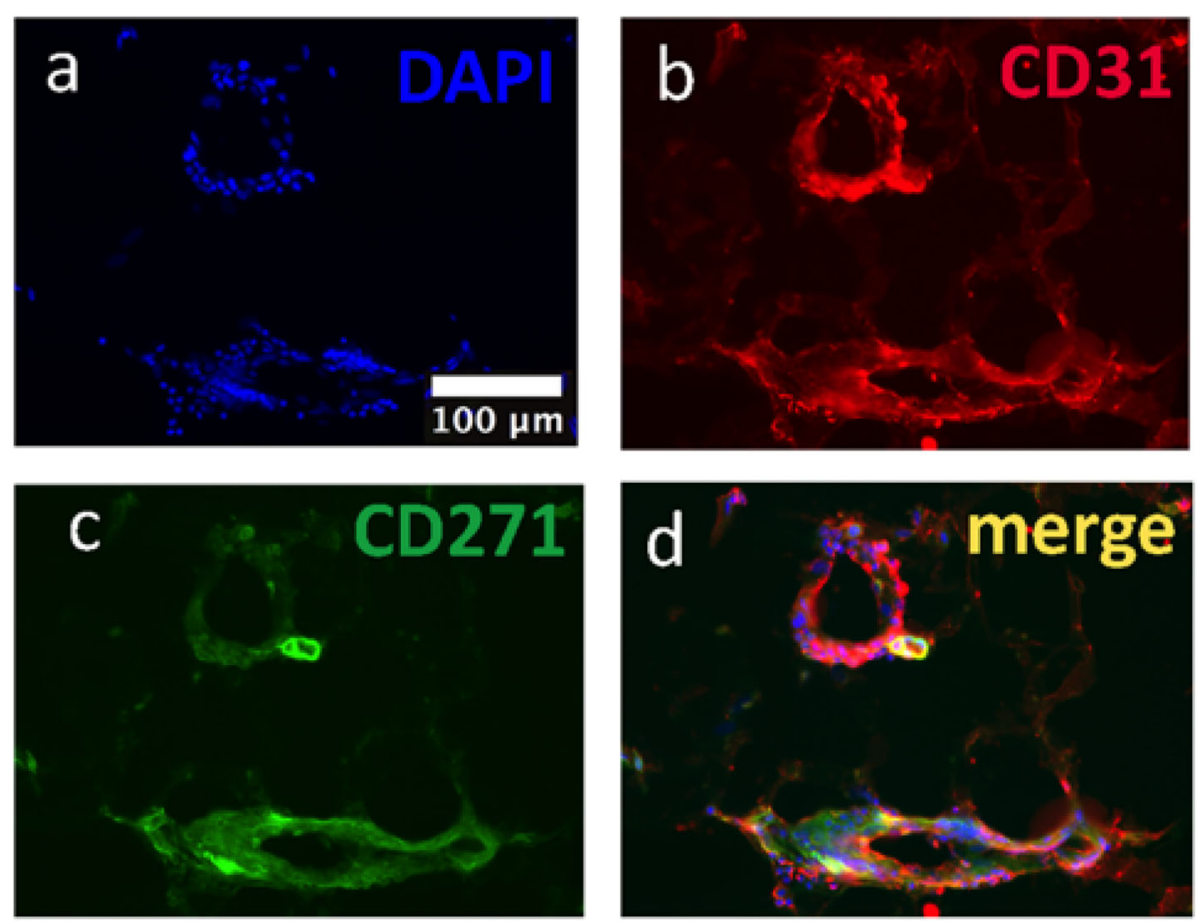

Fig. 1 Location of CD271+ AD-MSCs in vivo. Co-staining of fixed human adipose tissue for cell nuclei (a), CD31 (b), and CD271 (c) revealed colocalisation of CD271+ cells with CD31+ cells (d), indicating that CD271 cells are mostly localised around vascular structures

Sorting of human SVF using MACS successfully purified a CD271+ population as measured by flow analysis (Fig. 2a). Patients were consistent in the initial proportion of CD271+ AD-MSCs within SVF (average $20.6 \pm$ $1.5 \%, n=14)$, and sorting reliably produced a CD271+ population of average $86.6 \%$ purity $( \pm 1.3 \%, n=14)$. The sorted CD271- population contained an average of $14.5 \%$ CD271+ cells $( \pm 1.6 \%, n=14)$. Two-way ANOVA analysis with Tukey's multiple comparisons test revealed that all three groups were significantly different at the $p<0.0001$ level.

CD271 expression, as measured by flow cytometry, decreased in both sorted populations following cell culture (Fig. 2b). At passage 1, expression in CD271+ cells had dropped to just $25.9 \%( \pm 8.3 \%)$, which was not significantly different from CD271 expression in passage 1 CD271- cells (12.9 $\pm 5.1 \%)$. Expression continued to drop during subsequent culture, reaching a baseline of around 5\% expression in both populations at passage 6 $(n=4)$.

In passage 0 cells, immediately following sorting, typical stem cell markers were higher in CD271+ AD-MSCs (Fig. 2c-e). CD45 was present in low levels in both groups (Fig. 2c), but was significantly lower in CD271+ cells compared to CD271- cells $(4.4 \pm 0.9 \%$ vs. $17.3 \pm$ $1.1 \%, p<0.0001, n=8)$. CD90 was present in significantly higher levels in CD271+ cells compared to CD271 - cells (Fig. 2d, $91.9 \pm 1.8 \%$ vs. $70.4 \pm 4.9 \%, p=0.0011$, $n=8)$. CD271+ cells contained a significantly higher proportion of CD45-/CD90+ cells compared to CD271cells (Fig. 2 e, $86.8 \pm 2.7 \%$ vs. $56.2 \pm 6.2 \%, p=0.0005, n=$ 8).

RNA sequencing revealed significant changes in gene expression between CD271+ and CD271- AD-MSCs (Fig. 3). Principal component analysis confirmed that variation between sorted groups was greater than variation between patients (31\% vs. $23 \%$ variation, Fig. 3a). Genes of interest are highlighted in Fig. 3b, which displays all genes expressed with greater than 1.5 -fold difference in the CD271+ population compared to the CD271- population.

The eight selected genes of interest are also displayed in Table 1 with their respective fold changes and degrees of statistical significance. Angiogenic genes ANGPT2 and HGF were more than doubled in expression in CD271+ ADMSCs compared to the CD271- population, while angiogenic genes VEGFA and ANGPT1 showed a less significant trend towards higher expression. Inflammatory genes IL10RA, IL1B, and TNF were all reduced in CD271+ cells compared to CD271- AD-MSCs. WNT2, implicated in oncogenesis, was expressed lower in CD271+ cells.

Reactome analysis indicated that gene pathways associated with the immune system, extracellular matrix organisation, chemokine receptors, and IL10 signalling were most different between the CD271+ and CD271AD-MSC populations (Fig. 3c). 

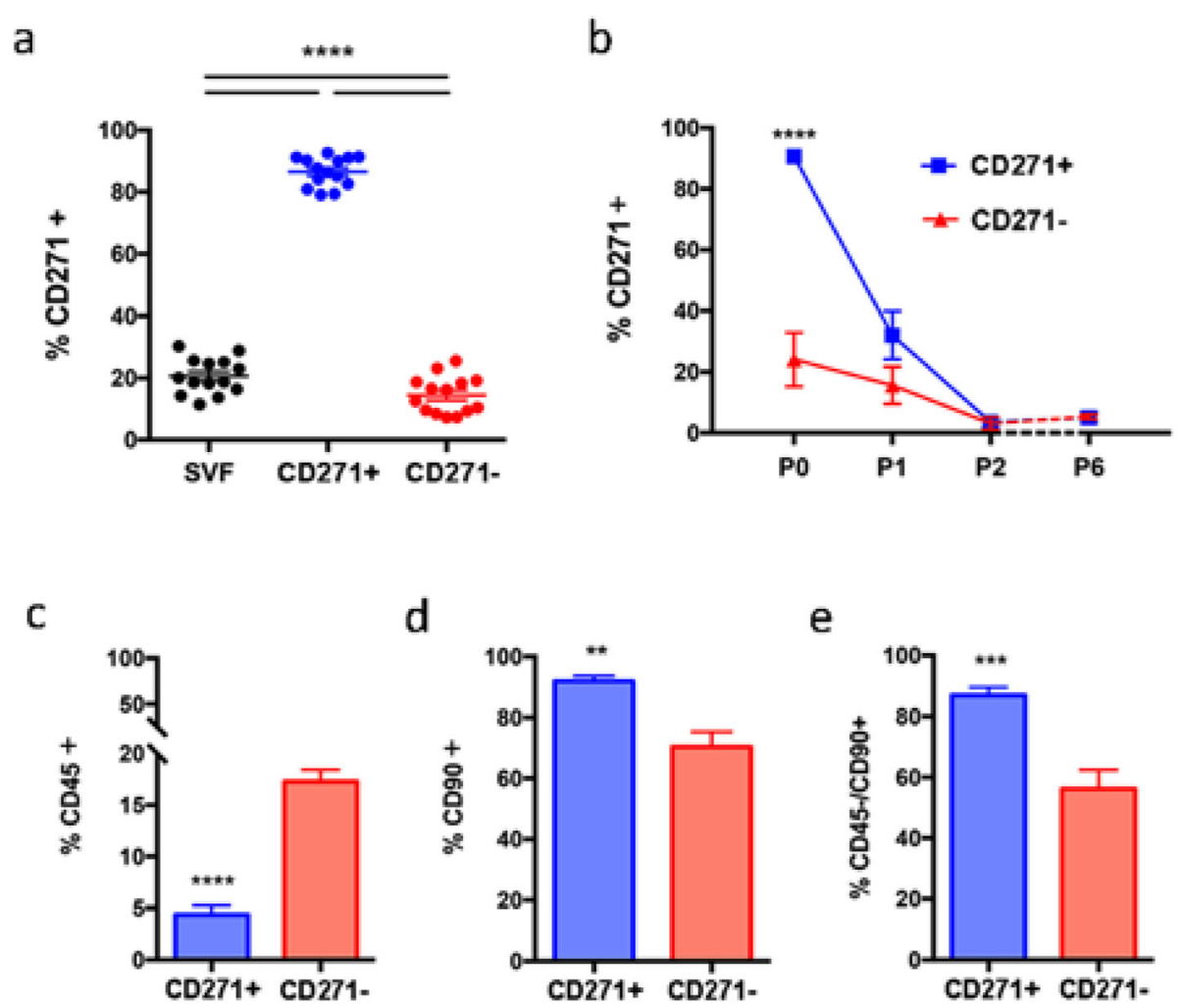

Fig. 2 Surface marker expression of AD-MSCs sorted for CD271. AD-MSCs sorted for CD271 were analysed for expression of surface markers using flow cytometry immediately following sorting, and during several passages of cell culture. Expression of CD271 was consistently high in the CD271 purified population (CD271+), compared to the negative sort (CD271-) and the unsorted SVF $(\mathbf{a}, n=14)$. During cell culture, CD271 expression rapidly decreased in both CD271+ and CD271- populations $(\mathbf{b}, n=4)$. CD45 expression was significantly lower in CD271+ AD-MSCs $(\mathbf{c}, n=8)$, and CD90 expression significantly higher $(\mathbf{d}, n=8)$. The number of CD45-/CD90+ cells was also higher in CD271+ AD-MSCs $(\mathbf{e}, n=8)$

Supplemental tables show genes with the highest fold increases and decreases between CD271+ and CD271AD-MSCs (Supplemental Tables 2 \& 3). Notably, expression of NGFR (CD271) is significantly increased by over fourfold in CD271+ cells $(p=4.3 \mathrm{E}-51)$, as another confirmation of the effectiveness of the technique. Supplemental Table 4 shows more detailed reactome pathway data.

Findings from RNA sequencing analyses were validated using real-time qPCR, which confirmed changes in genes of interest between CD271+ and CD271- AD-MSCs (Fig. 4). Although there was no significant difference in VEGFA expression (Fig. 4c, $1.00 \pm 0.12$ fold in CD271+ cells, $p=0.962, n=4)$, HGF was expressed higher in CD271+ cells compared to CD271- cells (Fig. 4d, $2.09 \pm$ 0.45 fold, $p<0.05, n=4$ ), and both ANGPT1 (Fig. 4a, $2.50 \pm 0.68$ fold, $p<0.05, n=4$ ) and ANGPT2 (Fig. 4b, $2.52 \pm 0.21$ fold, $p<0.05, n=4$ ) were expressed at higher levels, mirroring RNA sequencing results. Expression of both IL10RA and IL1B was lower in CD271+ cells compared to CD271- cells (Fig. 4e, $0.17 \pm 0.06$ fold, $p<0.05$, $n=4$; Fig. 4f, $0.23 \pm 0.06$ fold, $p<0.05, n=4$ ), and WNT2 levels were also decreased in CD271+ cells (Fig. 4h, $0.40 \pm$
0.12 fold, $p<0.05, n=4)$, again mirroring the RNA sequencing results. Levels of TNF were not significantly changed in this analysis (Fig. 4g, $1.42 \pm 0.23$ fold, $p=$ $0.066, n=4)$.

\section{HUVEC tubule formation assay with CD271+ AD-MSCs}

RFP-labelled HUVECs and CFSE-stained CD271+ ADMSCs were grown together for $16 \mathrm{~h}$, and fluorescence microscopy images were recorded to observe the potential involvement of CD271+ AD-MSCs within vascular networks (Fig. 5a-d). Merged imaging shows that CD271+ cells closely associated with tubules (Fig. 5d).

Unstained HUVECs were then grown with unstained CD271+ and CD271- AD-MSCs for $16 \mathrm{~h}$, and characteristics of the resultant tubule network were measured using light microscopy (Fig. $5 \mathrm{e}-\mathrm{g}$ ). Analysis of average tubule length showed that tubule length was slightly shorter when HUVECs were grown with CD271+ ADMSCs, compared to CD271- AD-MSCs $(0.86 \pm 0.03$ vs. $0.92 \pm 0.04$, normalised to HUVEC-only control, $p=$ 0.336, $n=6$, Fig. 5e). HUVECs grown with CD271+ ADMSCs were also significantly shorter than HUVECs grown alone $(0.86 \pm 0.03$ vs. $0.99 \pm 0.01$, normalised to 


\section{a}

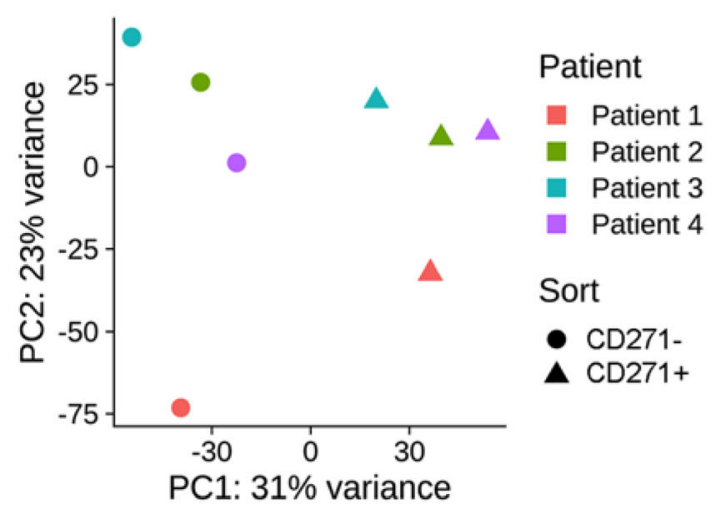

b

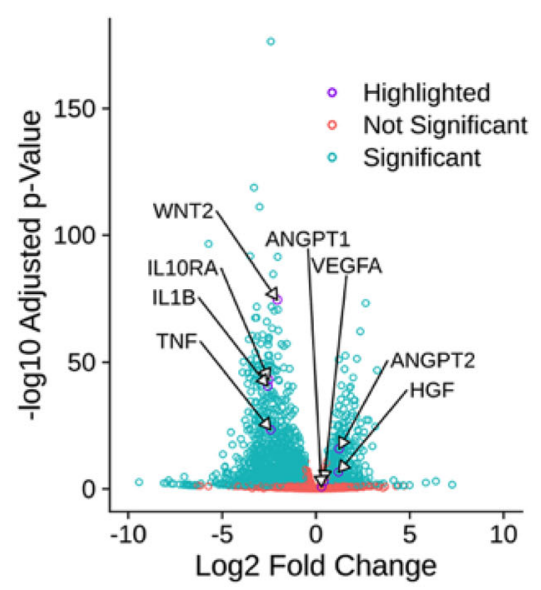

C

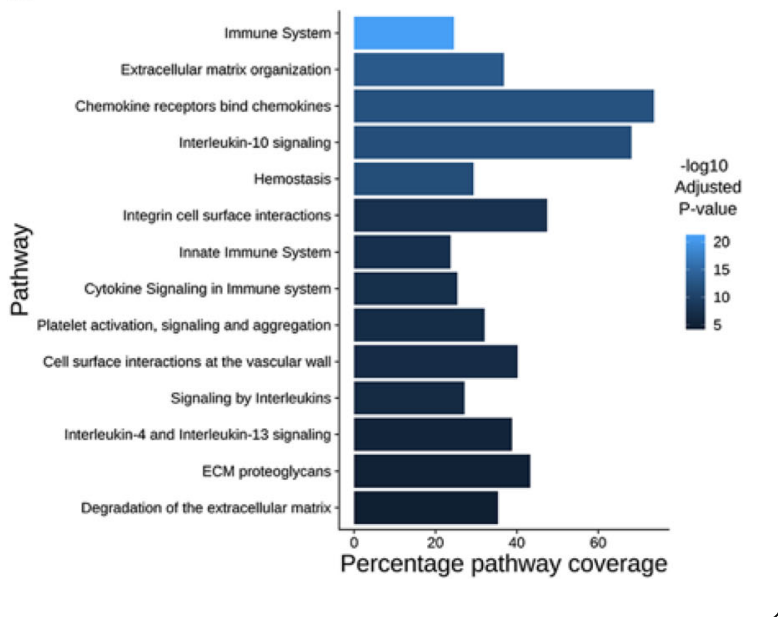

Fig. 3 RNA sequencing of CD271+ AD-MSCs. Principal component analysis (a) shows the degrees of variance between patients and between CD271+ and CD271- AD-MSCs. The volcano plot (b) shows all genes that changed by more than 1.5 fold in the CD271+ AD-MSCs compared to CD271- AD-MSCs. Significance is indicated in blue (significant, $p<0.05$ ) and red (non-significant, $p>0.05$ ). Data is represented as fold change in expression on the $x$-axis (in log2, with increases on the right hand side of the central ' 0 ,' and decreases on the left hand side of the central ' 0. '), against significance as a $p$ value on the $y$-axis (in - $\log 10$ ). Reactome analysis (c) indicates pathways of interest that are significantly altered in the CD271+ AD-MSCs compared to CD271- AD-MSCs

HUVEC-only control, $p=0.013, \mathrm{n}=6$, Fig. 5e). There were no significant differences in the number of tubules per field of view between groups, although HUVECs grown with CD271+ AD-MSCs tended to produce more tubules than when grown with CD271- AD-MSCs $(1.17 \pm 0.17$ vs. $0.83 \pm 0.11$, normalised to HUVEC-only control, $p=0.185, \mathrm{n}=6$, Fig. 5f). Although there was no significant difference in the average number of anastomoses per field of view between groups, HUVECs grown with CD271+ AD-MSCs had nearly double the number of anastomoses compared to those grown with CD271AD-MSCs $(1.75 \pm 0.60$ vs. $0.93 \pm 0.29$, normalised to HUVEC-only control, $p=0.321, n=6$, Fig. $5 \mathrm{~g}$ ).

\section{Co-culture of CD271+ AD-MSCs with AT}

Following 15 days of indirect co-culture between AD-MSCs and ex-vivo AT, levels of angiogenic protein in the media (ELISA) and expression of angiogenic genes in the ADMSCs (real-time qPCR) were measured. HGF and VEGFA protein levels and gene expression showed high inter-patient variability, and no clear pattern emerged. These results can be seen in Supplemental Figures 1 and 2.

ANGPT1 protein levels (Fig. 6, left column) showed a consistent pattern within patients (a) and (b). In these patients, ANGPT1 levels were highest in CD271+ cells grown with AT, a significant increase compared to CD271- cells grown with adipose tissue. Patient (a) expressed ANGPT1 at $607.6 \pm 16.3 \mathrm{pg} / \mathrm{ml}$ in CD271+ AD-MSCs grown with AT, compared to $465.7 \pm 14.4 \mathrm{pg} /$ $\mathrm{ml}$ in CD271- AD-MSCs grown with AT $(p<0.0001)$, with both of these groups expressing higher levels than in AT alone $(268.8 \pm 6.1 \mathrm{pg} / \mathrm{ml})$. In patient (b), not only were ANGPT1 levels significantly higher in CD271+ AD-MSCs with AT $(313.4 \pm 18.9 \mathrm{pg} / \mathrm{ml})$ compared to CD271- AD-MSCs with AT $(208.1 \pm 7.2 \mathrm{pg} / \mathrm{ml}, p=$ $0.0002)$ and AT alone $(176.7 \pm 10.1 \mathrm{pg} / \mathrm{ml}, p<0.0001)$, but CD271+ AD-MSCs grown in the absence of AT also produced higher levels of ANGPT1 than CD271- ADMSCs grown in the absence of AT $(258.3 \pm 11.0 \mathrm{pg} / \mathrm{ml}$ compared to $184.6 \pm 6.0 \mathrm{pg} / \mathrm{ml}, p=0.0037)$. In patient (c), there was no significant difference between any group, with ANGPT1 levels between 200 and $300 \mathrm{pg} / \mathrm{ml}$. 
Table 1 Expression changes in genes of interest in CD271+ AD-MSCs. Expression changes are presented as fold change in the CD271+ population compared to the CD271- population, by RNA sequencing analysis

\begin{tabular}{llll}
\hline Gene & Fold change & Adjusted $\boldsymbol{p}$ value & Gene function \\
\hline ANGPT1 & 1.22 & 0.156 & Vascular development and angiogenesis \\
ANGPT2 & 2.36 & $1.42 \mathrm{E}-16$ & Vascular remodelling, angiogenesis in combination with VEGF \\
VEGFA & 1.34 & 0.00120 & Vascular growth factor \\
HGF & 2.31 & $2.27 \mathrm{E}-07$ & Angiogenic growth factor \\
IL10RA & 0.18 & $7.55 \mathrm{E}-44$ & Inflammatory receptor \\
IL1B & 0.17 & $2.36 \mathrm{E}-41$ & Key cytokine in adipose tissue \\
TNF & 0.19 & $4.23 \mathrm{E}-24$ & Activates M1 macrophages in adipose tissue \\
WNT2 & 0.24 & $2.39 \mathrm{E}-75$ & Oncogenic gene
\end{tabular}

ANGPT1 angiopoietin 1, ANGPT2 angiopoietin 2, VEGFA vascular endothelial growth factor $\mathrm{A}$, HGF hepatocyte growth factor, IL10RA interleukin 10 receptor A, IL1B interleukin 1B, TNF tumour necrosis factor, WNT2 Wingless-type MMTV integration site family, member 2

In patient (d), all groups produced ANGPT1 levels around $300 \mathrm{pg} / \mathrm{ml}$, except for CD271- AD-MSCs grown with AT, which contained ANGPT1 at $357.3 \pm 18.3 \mathrm{pg} /$ $\mathrm{ml}$ (significant at $p<0.05$ compared to CD271+ ADMSCs with AT and AT alone).

ANGPT1 gene expression showed the most consistent pattern between patients for any of the angiogenic genes
(Fig. 6, right column). When grown with AT, CD271+ AD-MSCs showed significantly higher ANGPT1 expression compared to CD271- AD-MSCs in patients (a), (b), and (d). This increase was $1.22 \pm 0.02$ fold in patient (a), $2.90 \pm 0.04$ fold in patient (b), and $1.36 \pm 0.04$ fold in patient (d), compared to CD271- AD-MSCs (1 fold in all cases, $p<0.001$ in all cases). In addition, patient (b) a

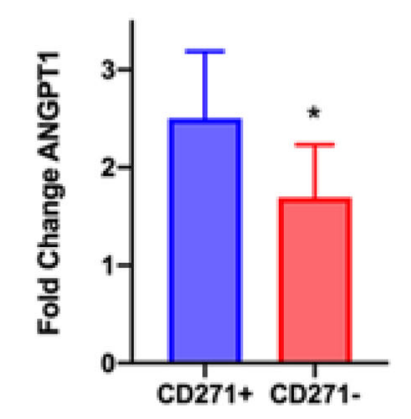

e

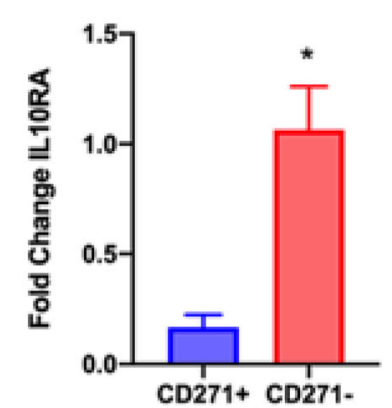

b

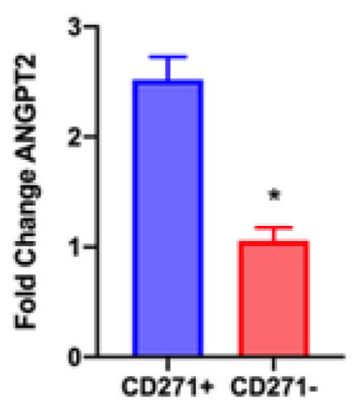

f

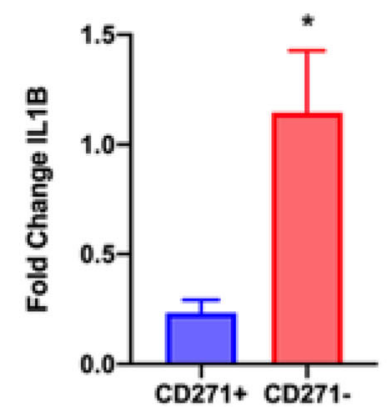

C

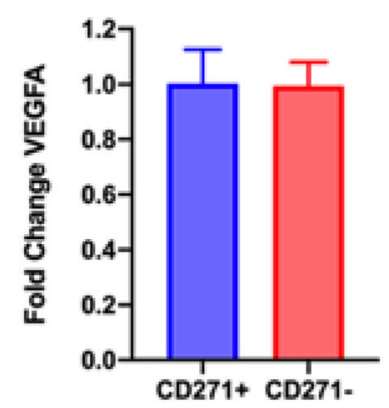

g

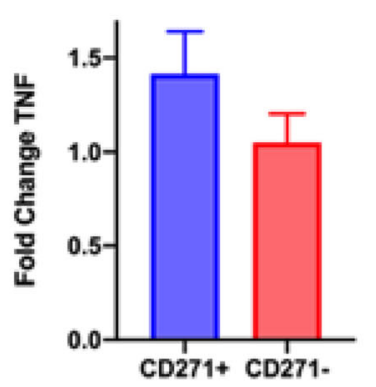

d

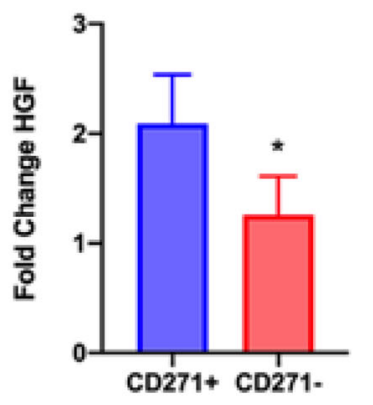

h

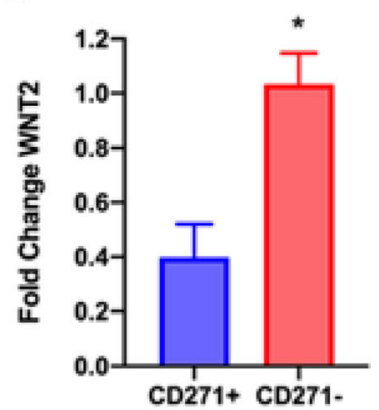

Fig. 4 Real-time qPCR analysis of CD271+ AD-MSCs. Analysis of RNA expression in AD-MSCs sorted for CD271, for angiogenic genes (a-d) and inflammatory/oncogenic genes (e-h). ANGPT1, angiopoietin 1; ANGPT2, angiopoietin 2; VEGFA, vascular endothelial growth factor A; HGF, hepatocyte growth factor; IL10RA, interleukin 10 receptor A; IL1B, interleukin 1B; TNF, tumour necrosis factor; WNT2, Wingless-type MMTV integration site family, member 2. The $\Delta \Delta C T$ method was used for analysis: all data is normalised to $18 \mathrm{~s}$, and to the between-patients average of CD271- AD-MSCs. $n=4$ 

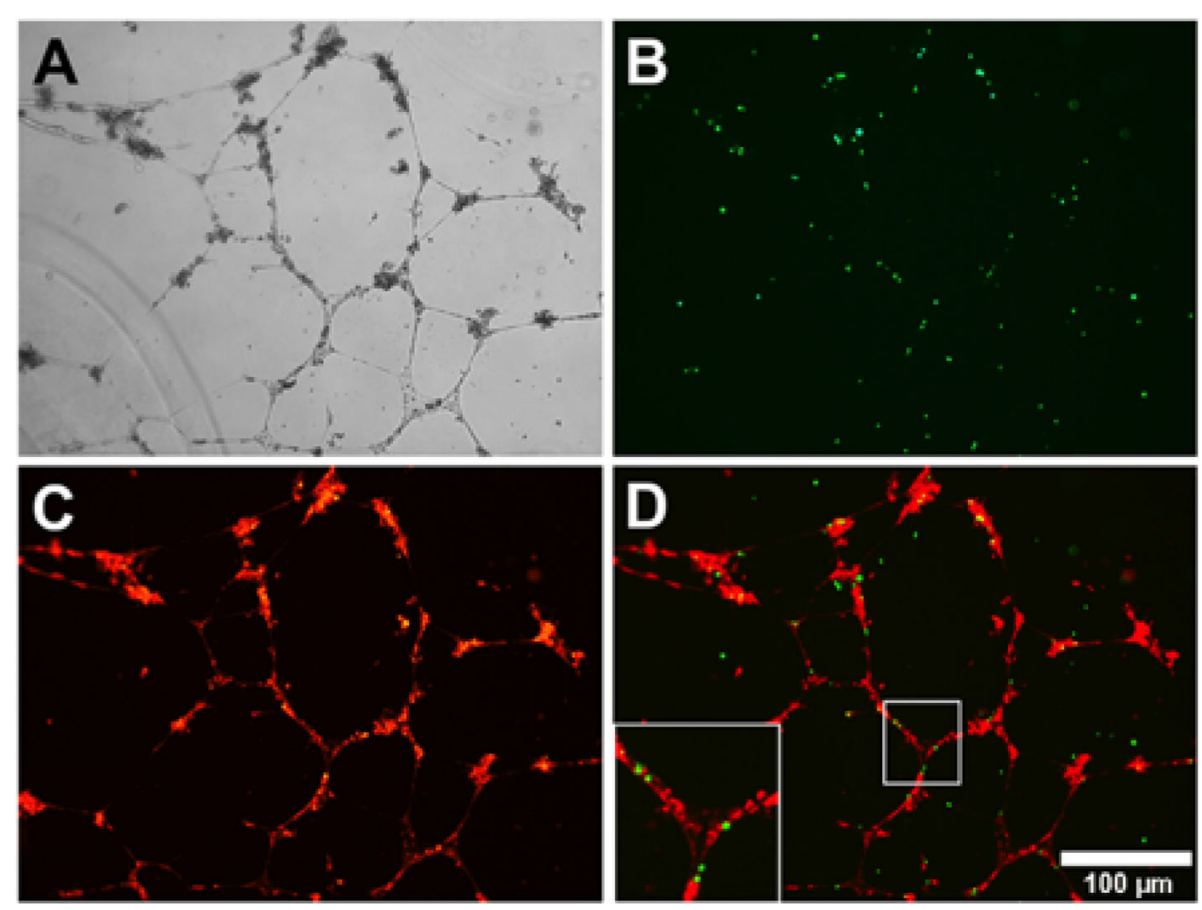

e

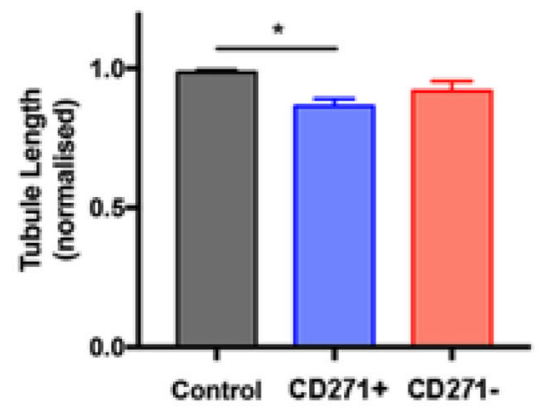

f

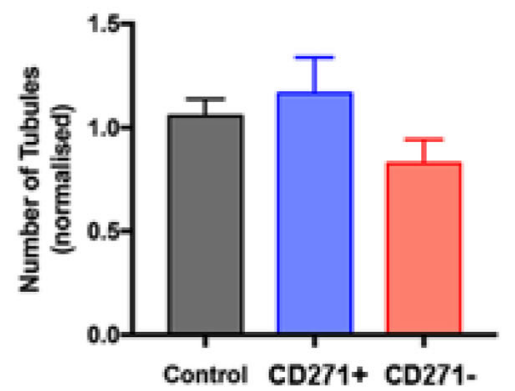

g

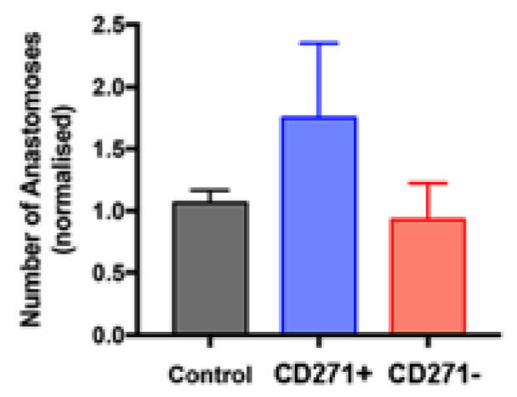

Fig. 5 Tubule formation analysis of HUVECs grown with CD271+ AD-MSCs. CD271+ AD-MSCs were grown with HUVECs for 16 h. Light microscopy reveals tubule formation (a). Staining the CD271+ AD-MSCS with CFSE (b) co-cultured with RFP-HUVECs (c) revealed co-localisation along the tubules (d, inset). Average tubule length, average number of tubules, and average number of anastomoses were measured after growing HUVECs with either CD271+ AD-MSCs or CD271- AD-MSCs, and data was normalised to HUVEC-only control $(\mathbf{e}-\mathbf{g}) . n=6$ 


\section{Protein}
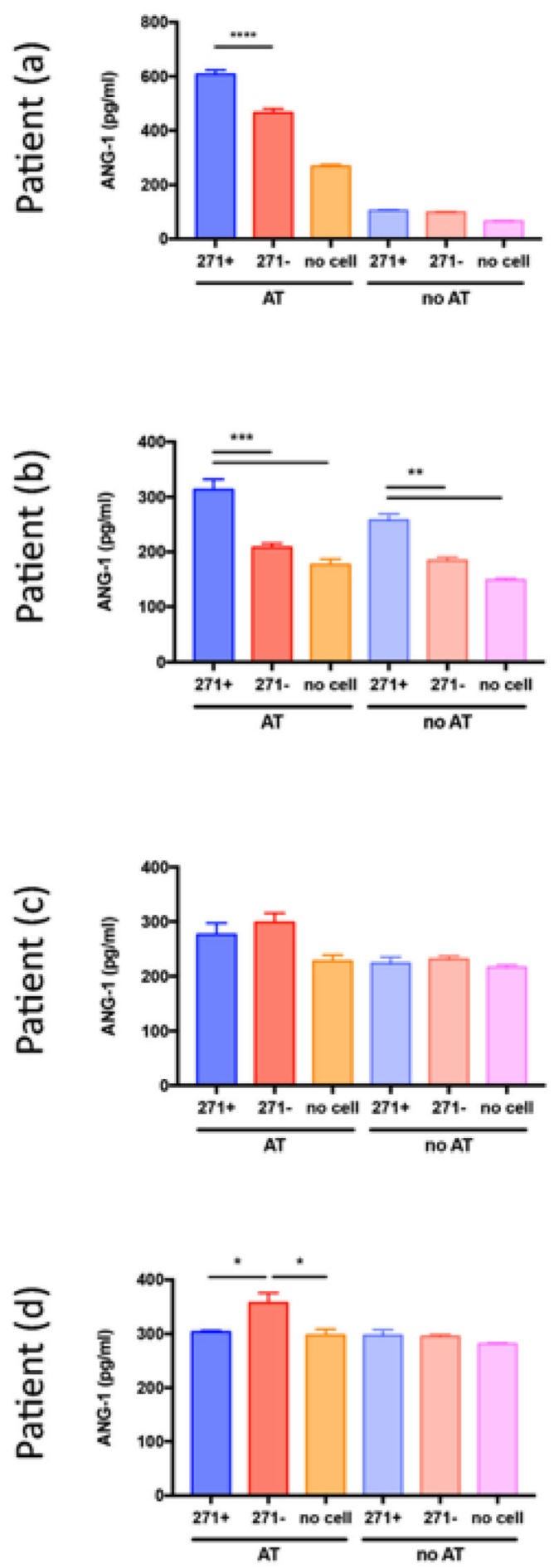

RNA
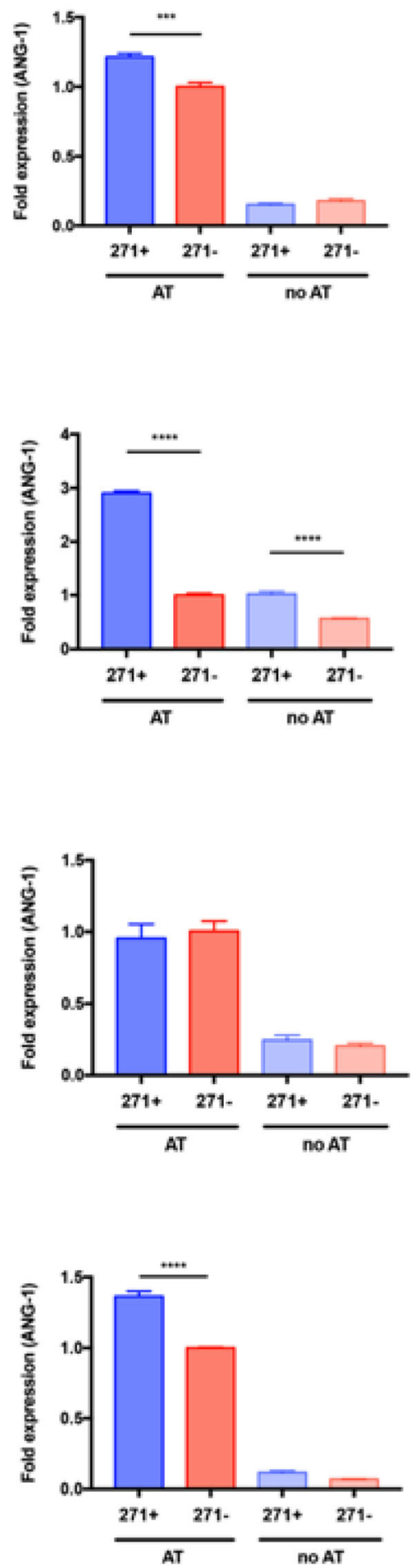

Fig. 6 Effect of CD271+ AD-MSCs and AT co-culture on ANGPT1. Both CD271+ and CD271- AD-MSCs were grown with AT, or without AT, for 15 days. Following this, protein levels of ANGPT1 in the co-culture media were measured by ELISA (left column), and RNA levels of ANGPT1 in the AD-MSCs were measured by real-time qPCR (right column). Individual patients are displayed due to high inter-patient variability. $n=3$ for each patient (experimental replicate) 
showed a significantly higher expression of ANGPT1 in CD271+ AD-MSCs in the absence of tissue compared to CD271- AD-MSCs $(1.02 \pm 0.04$ fold compared to $0.57 \pm$ 0.01 fold, $p<0.0001$ ). Patient (c) displayed no significant differences between cell sorts.

\section{Discussion}

CD271+ cells were confirmed present in human AT by immunocytochemistry and found to colocalise with vascular structures (stained with CD31), confirming previous literature that suggests CD271+ AD-MSCs exist in the adventitial niche of vasculature in human AT [21].

Magnetic sorting enriched the CD271 population from around 20\% in unsorted SVF, to over $85 \%$ in CD271+ AD-MSCs. This is comparable to other groups who have achieved similar high purities with MACS: Zhang et al. (2015) produced Kupffer cells of purity 95.7\% from mouse livers, after a double MACS sort for F4/80+ and CD11ccells, and Quirici et al. produced a CD271+ population from human AD-MSCs with $88.5 \%$ purity by MACS sorting $[14,22]$. This is despite prior claims that MACS produces significantly lower purities than FACS [23].

Depletion of CD271 from the negative sort was not as effective, with around 15\% expression remaining in CD271- AD-MSCs. There could be a number of reasons for this bleed-through of CD271+ cells into the CD271population-it is possible that not all CD271+ cells were stained by the microbead antibodies, or that CD271 expression was too low for antibody attachment while being high enough for detection in flow cytometry; rigorous methodological tests would have to be performed to provide an answer. However, for the purposes of this study, it was more pertinent to compare the characteristics and behaviour of the CD271+ population with SVF, rather than an entirely CD271-depleted population. Hence, the CD271- population could be considered practically very similar to the unsorted SVF, with the benefit of having been exposed to the same sorting procedure as the CD271+ population.

The finding that $20 \%$ of SVF is composed of CD271+ cells differs from some other literature. Quirici and colleagues found that only $4.4 \pm 6.3 \%$ of human SVF was composed of CD271+ cells [14], and a team led by Busser found that $11 \pm 4 \%$ of human SVF was CD271+ [16]. However, a recent study by Beckencamp showed a more similar purity of $19.6 \%$ of CD271+ cells in SVF [15]. This range in CD271+ purity is likely due to differences in experimental methodology; most markedly, among these three studies the extraction of SVF from AT was highly variable, with collagenase digestion times varying from 30 to $60 \mathrm{~min}$, and centrifugation speeds varying from 500 to $1200 \mathrm{~g}$. The latter two studies (which found higher purities of CD271) used lower centrifugation speeds, while our study used a much lower centrifugation speed $(160 g)$. It is likely that as groups further optimise the methodology of SVF extraction, cell survival will improve, making purity evaluations more accurate.

CD271 expression decreased dramatically in the CD271+ population following cell culture. After one passage, the proportion of cells expressing CD271 was reduced from over $80 \%$ to below $40 \%$. This is consistent with other studies that show significant changes in ADMSC phenotype following culture, such as reductions in haematopoietic and inflammatory markers and increases in stromal markers [24-26], and was justification for this study limiting its investigation to passage 0 cells.

CD271+ AD-MSCs were more likely to be CD90+/ CD45- compared to both CD271- AD-MSCs and unsorted SVF. As CD90 is a typical marker of a stem cell phenotype, and CD45 is a haematopoietic marker, the fact that CD271+ AD-MSCs were more likely to be CD90+ and CD45- highlights that CD271 is a marker for a more stem cell-like population [27].

RNA sequencing analysis revealed a number of important genetic differences between CD271+ and CD271AD-MSCs. Notably, principal component analysis revealed that genetic variation between CD271+ and CD271 - populations was more significant than between patients. Several hundred genes were significantly altered between the two populations, most pertinently in reactome pathways associated with inflammation and angiogenesis.

Angiogenic genes ANGPT1, ANGPT2, HGF, and VEGFA were expressed at higher levels in CD271+ ADMSCs, as measured by RNA sequencing. Real-time qPCR confirmed this, with the exception of VEGFA which was not changed significantly. As HGF is a crucial factor in tissue development and angiogenesis [28], and the angiopoietin family are important angiogenic factors in adipose tissue development and remodelling [29, 30], this finding is a good indicator of the proangiogenic potential of the CD271+ population.

The differences between ANGPT1 and ANGPT2 expression are of particular interest. Both these proteins act on the Tie2 receptor, activation of which leads to angiogenesis and anti-inflammation [29]. ANGPT1 is released by mature adipocytes and AD-MSCs, while ANGPT2 is known to be produced by vascular endothelial cells [29]. ANGPT1 activation of the Tie2 receptor leads to an increase in vascular endothelial cell density and can improve wound healing [30], while ANGPT2 has differing effects depending on VEGF presence. In low-VEGF environments, ANGPT2 inhibits the Tie2 receptor and promotes cell death, while in high-VEGF environments, ANGPT2 activates the Tie2 receptor in a unique pathway that produces vascular plasticity and prompts capillary growth [31]. As such, together they are involved in a system that regulates vascular 
remodelling and angiogenesis in AT (see Supplemental Figure 3).

The fact that RNA sequencing analysis shows significantly higher ANGPT2 expression in CD271+ AD-MSCs suggests that these cells are primed to influence vascular remodelling. Additionally, since ANGPT1 is mainly released by adipocytes, it is not surprising that RNA sequencing of sorted AD-MSCs revealed significant difference in ANGPT2 expression but no significant difference in ANGPT1 expression. Accordingly, later co-culture analysis revealed significantly higher ANGPT1 levels in CD271+ co-culture media, where presumably adipocytes are prompted to release ANGPT1 by CD271+ AD-MSCs.

Although sorted SVF cells have previously been demonstrated to possess proangiogenic properties [32], our findings display proangiogenic potential within an ADMSC subpopulation sorted without laboratory culture or manipulation. This is important for the potential clinical translation of AD-MSC subpopulation selection.

In addition to the proangiogenic potential we have seen in this CD271+ AD-MSC population, reactome analysis revealed that inflammatory pathways were most significantly changed between CD271+ and CD271AD-MSCs, suggesting that CD271+ AD-MSCs have anti-inflammatory potential. RNA sequencing revealed that inflammatory genes IL10RA, IL1B, and TNF were significantly downregulated in CD271+ AD-MSCs, which was confirmed by real-time qPCR analysis for IL10RA and IL1B. IL10RA is a key inflammatory receptor, and its overexpression is associated with kidney graft atrophy and fibrosis [33]. IL1B is a key cytokine involved in the inflammatory response to injury in adipose tissue [34], and TNF encourages the activation of M1 macrophages in adipose tissue [35].

Additionally, oncogenic gene WNT2 was expressed several times lower in CD271+ cells compared to CD271- ADMSCs: inhibitors of WNT2 are considered prime anti-cancer treatments [36]. A reduction in oncogenic factor expression within cellular therapies is particularly desirable for fat grafting treatments following mastectomies, where tissues may be especially susceptible to tumorigenesis.

In the HUVEC tubule formation assay, it was clear from CFSE/RFP co-staining that CD271+ AD-MSCs grew in close proximity with the pseudo-vascular networks. This complements our in vivo findings of CD271+ cells located near vascular structures in AT. Additionally, CD271+ AD-MSCs appeared to produce tubule networks of greater complexity (more tubules and more anastomoses) suggesting that this population would be more therapeutically beneficial in devascularised tissues-as smaller, more complex networks arguably indicate more effective microvascular growth, more accurately resembling capillary beds seen in vivo [37].
Growth factors present in Matrigel can potentially influence the results of HUVEC tubule formation assays, and this could have masked changes influenced by CD271+ AD-MSCs. To address this, the HUVEC experiments were also performed with growth factor-depleted Matrigel and the same results were observed.

Analysis of angiogenic protein levels and gene expression was performed in an AD-MSC/AT co-culture model, in order to understand how AD-MSCs and AT react to being in the same environment; both in terms of changes in AD-MSC transcription and the combined protein secretion of both AD-MSCs and AT. The analysis revealed high inter-patient variability, requiring a separation of individual patients to interpret the effects that co-culture had on protein levels and gene expression. Most notably, when CD271+ AD-MSCs were grown with AT, ANGPT1 expression was consistently and significantly higher in these cells compared to CD271- AD-MSCs, in three out of four patients. This higher expression also appeared to prompt higher ANGPT1 protein production by both $\mathrm{AT}$ and $\mathrm{AD}$ MSCs, in two of the four patients analysed. In one patient, this increase in ANGPT1 protein was more than cumulative; in other words, AT and AD-MSCs were interacting in a way that prompted more ANGPT1 production than the combination of the amounts they produced on their own. Although the inter-patient variability in this analysis is a cause to question the general applicability of subpopulation selection in surgical techniques, overall these findings support the proangiogenic potential of CD271+ AD-MSCs.

The method of MACS sorting for CD271+ AD-MSCs precludes cell culture or phenotype manipulation and therefore has high potential for clinical translation. Although in vitro cell manipulation can show promising results [32, 38], it is more efficient and safe to avoid laboratory processing and differentiation of cells. MACS sorting of CD34+ cells is already utilised in the surgical treatment of haematological malignancies, so the technology is already translatable [39]. The within-surgery sorting of CD271+ AD-MSCs has great clinical potential as an autologous approach to improve the survival of adipose tissue, especially in large volume fat grafting during breast reconstruction.

\section{Conclusions}

This study demonstrates the proangiogenic potential of CD271+ AD-MSCs and presents this subpopulation as a preferable cellular therapy compared to the more commonly used SVF. Clinical application of cell subpopulation therapies is already established, and the introduction of CD271+ AD-MSCs in therapies such as fat grafting presents a unique and potentially highly translatable method for improving tissue engineering 
approaches. More work will be required to better understand the patient-to-patient variability in the characteristics of CD271+ AD-MSCs, and extensive in vivo experiments must be performed prior to clinical translation.

\section{Supplementary Information}

The online version contains supplementary material available at https:/doi. org/10.1186/s13287-021-02177-0

Additional file 1: Supplemental Figure 1. Effect of CD271+ AD-MSCS and AT co-culture on HGF, Supplemental.

Additional file 2: Supplemental Figure 2. Effect of CD271+ AD-MSCS and AT co-culture on VEGFA.

Additional file 3: Supplemental Figure 3. The dynamics of angiopoietin in adipose tissue.

Additional file 4: Supplemental Table 1. Primers used in real-time qPCR analysis. Supplemental Table 2. Greatest gene expression decreases in CD271+ sorted AD-MSCs compared to CD271- sorted ADMSCs. Supplemental Table 3. Greatest gene expression increases in CD271+ sorted AD-MSCs compared to CD271- sorted AD-MSCs. Supplemental Table 4. Full reactome pathway analysis results.

\section{Abbreviations}

AD-MSCs: Adipose tissue-derived mesenchymal stem cells; ANGP T1: Angiopoietin 1; ANGPT2: Angiopoietin 2; AT: Adipose tissue; BMMSCs: Bone marrow-derived mesenchymal stem cells;

CFSE: Carboxyfluorescein succinimidyl ester; ELISA: Enzyme-linked immunosorbent assay; FACS: Fluorescent-activated cell sorting: HGF: Hepatocyte growth factor; HUVECs: Human umbilical vascular endothelial cells; IL10RA: Interleukin 10 receptor A; IL1B: Interleukin 1B; LNGF R: Low affinity nerve growth factor receptor; MACS: Magnetic-activated cell sorting; NGFR: Nerve growth factor receptor; RFP: Red fluorescent protein; SVF: Stromal vascular fraction; TGF-B: Transforming growth factor beta; TNF: Tumour necrosis factor; VEGFA: Vascular endothelial growth factor A; WNT2: Wingless-type MMTV integration site family, member 2

\section{Acknowledgements}

We thank the Bioinformatics and Genomic Technologies Core Facilities at the University of Manchester for providing support with regard to RNA-seq. The authors also thank Mr. Jonathan Duncan and Miss Siobhan O'Ceallaigh (Consultant Plastic Surgeons) and their patients at Wythenshawe Hospital, Manchester University NHS Foundation Trust, for the donation of adipose tissue.

\section{Authors' contributions}

Conceptualisation, R.J.P.S., A.F., and A.J.R.; methodology, R.J.P.S., A.F., and A.J.R.; validation, A.J.R.; formal analysis, R.J.P.S., A.F., A.J.R., J.R.B., and J.S.; investigation, R.J.P.S., A.F., J.R.B., and J.S.; resources, A.J.R.; data curation, R.J.P.S., A.F., and J.S.; writing-original draft preparation, R.J.P.S. and A.F.; writing-review and editing, R.J.P.S., A.F., and A.J.R.; visualisation, R.J.P.S., A.F., A.J.R., and J.S.; supervision, A.J.R.; project administration, A.J.R.; funding acquisition, A.J.R. All authors have read and agreed to the final version of the manuscript.

\section{Funding}

This research was funded by The Scar Free Foundation through a BAAPS Research Award in memory of Dorothy Sainsbury. The Scar Free Foundation is the only medical research charity focused on scarring with the mission to achieve scar free healing within a generation. AF and AJR are supported by the Henry Lumley Trust and the Academy of Medical Sciences (AMS-SGCL7Reid).

\section{Availability of data and materials}

All data generated or analysed during this study are included in this published article [and its supplementary information files]. The data that support the findings of this study are openly available in ArrayExpress at http://www.ebi.ac.uk/arrayexpress/experiments/E-MTAB-8740.

\section{Ethics approval and consent to participate}

Abdominal adipose tissue was harvested from donor patients undergoing DIEP flap breast reconstruction at Wythenshawe Hospital, Manchester University NHS Foundation Trust, UK, after informed consent was granted. Ethics approval was given from the NHS Health Research Authority, National Research Ethics Service Committee South Central - Hampshire B: reference 13/SC/0499.

\section{Consent for publication}

Not applicable.

\section{Competing interests}

The authors declare that they have no competing interests.

\section{Author details}

${ }^{1}$ Blond Mclndoe Laboratories, Division of Cell Matrix Biology and Regenerative Medicine, School of Biological Sciences, Faculty of Biology, Medicine and Health, The University of Manchester, Manchester Academic Health Science Centre, Manchester M13 9PT, UK. Biosciences Institute, Faculty of Medical Sciences, Newcastle University, Newcastle Upon Tyne, UK. ${ }^{3}$ Department of Plastic Surgery \& Burns, Wythenshawe Hospital, Manchester University NHS Foundation Trust, Manchester Academic Health Science Centre, Manchester, UK.

Received: 21 October 2020 Accepted: 19 January 2021

Published online: 02 March 2021

\section{References}

1. Agha RA, Fowler AJ, Herlin C, et al. Use of autologous fat grafting for breast reconstruction: a systematic review with meta-analysis of oncological outcomes. J Plast Reconstr Aesthet Surg. 2015;68(2):143-61.

2. Rasmussen BS, Lykke Sorensen C, Vester-Glowinski PV, et al. Effect, feasibility, and clinical relevance of cell enrichment in large volume fat grafting: a systematic review. Aesthet Surg J. 2017;37(suppl_3):S46-58.

3. Nguyen A, Guo J, Banyard DA, et al. Stromal vascular fraction: a regenerative reality? Part 1: current concepts and review of the literature. J Plast Reconstr Aesthet Surg. 2016;69(2):170-9.

4. Rehman J, Traktuev D, Li J, et al. Secretion of angiogenic and antiapoptotic factors by human adipose stromal cells. Circulation. 2004;109(10):1292-8.

5. Kang $T$, Jones TM, Naddell $C$, et al. Adipose-derived stem cells induce angiogenesis via microvesicle transport of miRNA-31. Stem Cells Transl Med. 2016:5(4):440-50.

6. Dong Z, Peng Z, Chang Q, et al. The survival condition and immunoregulatory function of adipose stromal vascular fraction (SVF) in the early stage of nonvascularized adipose transplantation. PLoS One. 2013;8(11):e80364.

7. Cohen SR, Hewett $S$, Ross $L$, et al. Regenerative cells for facial surgery: biofilling and biocontouring. Aesthet Surg J. 2017;37(suppl_3):S16-32.

8. Chen $Y$, Zeng J, Cen L, et al. Multiple roles of the p75 neurotrophin receptor in the nervous system. J Int Med Res. 2009;37(2):281-8.

9. You S, Petrov T, Chung PH, et al. The expression of the low affinity nerve growth factor receptor in long-term denervated Schwann cells. Glia. 1997;20(2):87-100.

10. Quirici N, Soligo D, Bossolasco P, et al. Isolation of bone marrow mesenchymal stem cells by anti-nerve growth factor receptor antibodies. Exp Hematol. 2002;30(7):783-91.

11. Son MY, Choi H, Han YM, et al. Unveiling the critical role of REX1 in the regulation of human stem cell pluripotency. Stem Cells. 2013;31(11):2374-87.

12. Liu J, Sato $C$, Cerletti $M$, et al. Notch signaling in the regulation of stem cell self-renewal and differentiation. Curr Top Dev Biol. 2010;92:367-409.

13. Michalczyk K, Ziman M. Nestin structure and predicted function in cellular cytoskeletal organisation. Histol Histopathol. 2005;20(2):665-71.

14. Quirici N, Scavullo C, de Girolamo L, et al. Anti-L-NGFR and-CD34 monoclonal antibodies identify multipotent mesenchymal stem cells in human adipose tissue. Stem Cells Dev. 2010;19(6):915-25.

15. Beckenkamp LR, Souza LEB, Melo FUF, et al. Comparative characterization of CD271(+) and CD271(-) subpopulations of CD34(+) human adipose-derived stromal cells. J Cell Biochem. 2018:119(5):3873-84.

16. Busser $H$, Najar M, Raicevic $G$, et al. Isolation and characterization of human mesenchymal stromal cell subpopulations: comparison of bone marrow and adipose tissue. Stem Cells Dev. 2015;24(18):2142-57. 
17. Cuevas-Diaz Duran R, Gonzalez-Garza MT, Cardenas-Lopez A, et al. Agerelated yield of adipose-derived stem cells bearing the low-affinity nerve growth factor receptor. Stem Cells Int. 2013;2013:372164.

18. Young MD, Wakefield MJ, Smyth GK, et al. Gene ontology analysis for RNAseq: accounting for selection bias. Genome Biol. 2010;11(2):R14.

19. Fabregat $A$, Jupe $S$, Matthews $L$, et al. The reactome pathway knowledgebase. Nucleic Acids Res. 2018;46(D1):D649-55.

20. Anayama H, Fukuda R, Yamate J. Adipose progenitor cells reside among the mature adipocytes: morphological research using an organotypic culture system. Cell Biol Int. 2015;39(11):1288-98.

21. Yang $\mathrm{S}$, Eto $\mathrm{H}$, Kato $\mathrm{H}$, et al. Comparative characterization of stromal vascular cells derived from three types of vascular wall and adipose tissue. Tissue Eng A. 2013;19(23-24):2724-34.

22. Zhang C, Lu Y, Zhou H, et al. Acquiring Kupffer cells in mice using a MACSbased method. Transplant Proc. 2015:47(2):553-7.

23. Li Q, Zhang $X$, Peng $Y$, et al. Comparison of the sorting efficiency and influence on cell function between the sterile flow cytometry and immunomagnetic bead purification methods. Prep Biochem Biotechnol. 2013;43(2):197-206.

24. Yoshimura K, Shigeura T, Matsumoto D, et al. Characterization of freshly isolated and cultured cells derived from the fatty and fluid portions of liposuction aspirates. J Cell Physiol. 2006;208(1):64-76.

25. McIntosh K, Zvonic S, Garrett S, et al. The immunogenicity of human adipose-derived cells: temporal changes in vitro. Stem Cells. 2006;24(5): 1246-53.

26. Mitchell JB, Mclntosh K, Zvonic S, et al. Immunophenotype of human adipose-derived cells: temporal changes in stromal-associated and stem cell-associated markers. Stem Cells. 2006;24(2):376-85.

27. De Francesco F, Tirino V, Desiderio V, et al. Human CD34/CD90 ASCs are capable of growing as sphere clusters, producing high levels of VEGF and forming capillaries. PLoS One. 2009;4(8):e6537.

28. Nakamura T, Mizuno S. The discovery of hepatocyte growth factor (HGF) and its significance for cell biology, life sciences and clinical medicine. Proc J.jn Acad Ser B Phys Biol Sci. 2010;86(6):588-610.

29. Dallabrida SM, Zurakowski D, Shih SC, et al. Adipose tissue growth and regression are regulated by angiopoietin-1. Biochem Biophys Res Commun. 2003;311(3):563-71.

30. Jung YJ, Choi HJ, Lee JE, et al. The effects of designed angiopoietin-1 variant on lipid droplet diameter, vascular endothelial cell density and metabolic parameters in diabetic $\mathrm{db} / \mathrm{db}$ mice. Biochem Biophys Res Commun. 2012;420(3):498-504.

31. Lobov IB, Brooks PC, Lang RA. Angiopoietin-2 displays VEGF-dependent modulation of capillary structure and endothelial cell survival in vivo. Proc Natl Acad Sci U S A. 2002;99(17):11205-10

32. Planat-Benard V, Silvestre JS, Cousin B, et al. Plasticity of human adipose lineage cells toward endothelial cells: physiological and therapeutic perspectives [in eng]. Circulation. 2004;109(5):656-63.

33. Maluf DG, Mas VR, Archer KJ, et al. Molecular pathways involved in loss of kidney graft function with tubular atrophy and interstitial fibrosis. Mol Med. 2008; 14(5-6):276-85.

34. Saraf MK, Herndon DN, Porter C, et al. Morphological changes in subcutaneous white adipose tissue after severe burn injury. J Burn Care Res. 2016;37(2):e96-103.

35. Ait-Lounis A, Laraba-Djebari F. TNF-alpha modulates adipose macrophage polarization to M1 phenotype in response to scorpion venom. Inflamm Res. 2015;64(11):929-36.

36. Katoh M, Katoh M. WNT signaling pathway and stem cell signaling network Clin Cancer Res. 2007;13(14):4042-5.

37. Staton CA, Reed MW, Brown NJ. A critical analysis of current in vitro and in vivo angiogenesis assays. Int J Exp Pathol. 2009;90(3):195-221.

38. Desiderio V, De Francesco F, Schiraldi C, et al. Human Ng2+ adipose stem cells loaded in vivo on a new crosslinked hyaluronic acid-lys scaffold fabricate a skeletal muscle tissue. J Cell Physiol. 2013;228(8):1762-73.

39. Watts MJ, Somervaille TC, Ings SJ, et al. Variable product purity and functional capacity after CD34 selection: a direct comparison of the CliniMACS (v2.1) and Isolex 300i (v2.5) clinical scale devices. Br J Haematol. 2002;118(1):117-23.

\section{Publisher's Note}

Springer Nature remains neutral with regard to jurisdictional claims in published maps and institutional affiliations.

\section{Ready to submit your research? Choose BMC and benefit from:}

- fast, convenient online submission

- thorough peer review by experienced researchers in your field

- rapid publication on acceptance

- support for research data, including large and complex data types

- gold Open Access which fosters wider collaboration and increased citations

- maximum visibility for your research: over $100 \mathrm{M}$ website views per year

At BMC, research is always in progress.

Learn more biomedcentral.com/submissions 